\title{
Pentacene Active Channel Layers Prepared by Spin-Coating and Vacuum Evaporation Using Soluble Precursors for OFET Applications
}

\author{
Shizuyasu Ochiai, ${ }^{1}$ Kumar Palanisamy, ${ }^{1}$ Santhakumar Kannappan, ${ }^{1}$ and Paik-Kyun Shin ${ }^{2}$ \\ ${ }^{1}$ Department of Electrical Engineering, Aichi Institute of Technology, Toyota City 470-0392, Japan \\ ${ }^{2}$ School of Electrical Engineering, Inha University, 253 Yonghyun-gong, Nam-gu, Incheon 402-751, Republic of Korea \\ Correspondence should be addressed to Shizuyasu Ochiai, ochiai@aitech.ac.jp
}

Received 30 August 2012; Accepted 15 September 2012

Academic Editors: V. Kochereshko and L. Pusztai

Copyright (C) 2012 Shizuyasu Ochiai et al. This is an open access article distributed under the Creative Commons Attribution License, which permits unrestricted use, distribution, and reproduction in any medium, provided the original work is properly cited.

\begin{abstract}
Pentacene OFETs of bottom-gate/bottom-contact were fabricated with three types of pentacene organic semiconductors and cross linked Poly(4-vinylphenol) or polycarbonate as gate dielectric layer. Two different processes were used to prepare the pentacene active channel layers: (1) spin-coating on dielectric layer using two different soluble pentacene precursors of SAP and DMP; (2) vacuum evaporation on PC insulator. X-ray diffraction studies revealed coexistence of thin film and bulk phase of pentacene from SAP and thin film phase of pentacene from DMP precursors. The field effect mobility of $0.031 \mathrm{~cm}^{2} /$ Vs and threshold voltage of $-12.5 \mathrm{~V}$ was obtained from OFETs fabricated from SAP precursor, however, the pentacene OFETs from DMP under same preparation yielded high mobility of $0.09 \mathrm{~cm}^{2} / \mathrm{Vs}$ and threshold value decreased to $-5 \mathrm{~V}$. It reflects that the mixed phase films had carrier mobilities inferior to films consisting solely of single phase. For comparison, we have also fabricated pentacene OFETs by vacuum evaporation on polycarbonate as the gate dielectric and obtained charge carrier mobilities as large as $0.62 \mathrm{~cm}^{2} / \mathrm{Vs}$ and threshold voltage of $-8.5 \mathrm{~V}$. We demonstrated that the spin-coated pentacene using soluble pentacene precursors could be alternative process technology for low cost, large area and low temperature fabrication of OFETs.
\end{abstract}

\section{Introduction}

Organic electronics are receiving great attention as emergent technology because of their inherent capacity to realize different electronic devices on desirable and flexible substrates. Organic molecular semiconductors and polymers used as active elements in optoelectronic devices have attracted great deal of attention today. Various organic molecular semiconductors and polymers have now demonstrated performance, which makes them suitable for application in thinfilm-based devices via solution processes and cost-effective methods $[1,2]$. Organic field effect transistors (OFETs) paved a new way due to their application to flexible, low cost, and large area thin film devices for various kinds of sophisticated organic electronic devices such as organic light emitting diodes (OLEDs), organic photovoltaic cells (OPV), drivers for electronic papers (e-papers), radio frequency identification tag (RFID), flexible memory, and flat panel displays $[3,4]$. OFETs were organic active layers deposited upon gate dielectrics, for example, silicon dioxide $\left(\mathrm{SiO}_{2}\right)$ or polymer dielectrics fabricated by vacuum evaporation, chemical vapour deposition; hot wall epitaxy and spincoating have been reported to show good working performances [5]. To realize practical applications with appropriate organic-based transistors, choice of organic semiconductors with high carrier mobility and gate dielectric materials is important. The organic semiconductors structure at the interface with the dielectric layer is a crucial factor that has an impact on the field effect mobility in the OFETs [6].

To develop an appropriate organic semiconductor for OFETs, numerous studies have been carried out over the last decade on $p$-type organic semiconductor layers such as pentacene, polyacene derivatives, oligothiophene derivatives, and polythiophene derivatives. Among them 
pentacene, consisting of five fused benzene rings, is a highly promising candidate for application in OFETs. The advantages of pentacene are high air stability and good semiconducting behaviour [7]. It exhibits $p$-type transport due to high hole carrier mobilities. The field effect mobilities of pentacene OFETs prepared by various methods have been steadily increasing from 0.001 to $3 \mathrm{~cm}^{2} / \mathrm{Vs}[8,9]$. The main drawback of pentacene is that it is insoluble in common organic solvents at room temperature since the solubility of pentacene in ordinary organic solvents is extremely low. OFETs are aiming wide range of inexpensive applications alternative gate dielectrics, and low-cost fabrication methods are of interest, including solution-processable pentacene precursors $[10,11]$ and polymer gate dielectrics [3, 12], which can be deposited by spin-coating or spray-coating, rather than by vacuum deposition. Polymer gate dielectrics have some advantages [13] and are used as an alternative to conventional inorganic dielectrics.

The OFETs reported up to now revealed promising performances regarding field effect mobility $\left(\mu_{\mathrm{FE}}\right)$, threshold voltage $\left(V_{\text {th }}\right)$, and on/off current ratio $\left(I_{\text {on } / \text { off }}\right)$, currently dominating inorganic TFTs based on low temperature polysilicon (LTPS) semiconductors or metal-oxide semiconductors that show much higher level of performances which might not be likely accomplished for OFETs. Therefore, it is highly desirable for OFETs to be applied to flexible electronics, for which following preconditions should be met: (1) low temperature process technologies compatible to flexible substrates; (2) easy and low-cost process technologies for large area/mass production; (3) reliable encapsulation/passivation process technologies for commercial-level devices. In order to explore the above-mentioned tasks of OFETs for flexible electronics applications, we fabricated $p$-type pentacene OFETs using cross-linked poly(4-vinylphenol) (cPVP) gate insulating layer. For the $p$-type pentacene semiconductor preparation, a solution-based spin-coating process was carried out using two different soluble pentacene precursors of 13,6-N-Sulfinylacetamidopentacene (SAP) and 6,13-Dihydro-6,13-methanopentacene-15-one (DMP). The preparation methods of the solution, solution-processed thin films, and their characterization have been presented. Fieldeffect transistors formed with solution processed films by direct formation were fabricated. To evaluate the performance of devices, we have also fabricated pentacene OFETs by vacuum evaporation using polycarbonate (PC) as the gate dielectric layer. The obtained results show that organic gate dielectrics possess potentially inexpensive alternative to inorganic counterparts and good electrical performance.

\section{Experimental}

2.1. Materials and Dielectric Layer. Pentacene (98\%), poly(4-vinylphenol) (PVP, Mw-20000), poly(melamineco-formaldehyde) methylated (Mn-511), propylene glycol monomethyl ether acetate (PGMEA), soluble pentacene precursors of 13,6-N-Sulfinylacetamidopentacene (SAP, 97\%), and 6,13-Dihydro-6,13-methanopentacene-15-one (DMP, 97\%) were purchased from Sigma-Aldrich. Polycarbonate
(PC, FE-2000) was purchased from Mitsubishi EngineeringPlastic. The substrates used are commercially available Pyrex glass. The substrates were cleaned with acetone, isopropyl alcohol, and methyl alcohol by ultrasonication for $15 \mathrm{~min}$ each. At each cleaning step, the substrates were rinsed with deionised water for $5 \mathrm{~min}$ in an ultrasonicator and purged with nitrogen gas. The cleaned substrates were dried at $100^{\circ} \mathrm{C}$ in a vacuum oven for at least $30 \mathrm{~min}$ before use. Pentacene for the active layer formation was used without further purification. PVP has been frequently used as dielectric layer because of its higher device performance but hydroxyl groups in the PVP dielectrics may easily react with water and mobile ions that deteriorate device function. Hence, to reduce the hydroxyl groups PVP has been cross-linking with curing agents by both thermal and photochemical treatments. Standard solutions of PVP were prepared with poly(melamine-co-formaldehyde) methylated as a cross-linked agent (CLA) in a ration of $11: 3$ (PVP : CLA) by weight in PGMEA followed by prebake at $100^{\circ} \mathrm{C}$ for $10 \mathrm{~min}$ and main bake in $\mathrm{N}_{2}$ atmosphere at $200^{\circ} \mathrm{C}$ for $40 \mathrm{~min}$; this polymer layer started to form cross-linked structure. Additionally, another cross-linkable PVP layer was prepared by UV irradiation. The cPVP was prepared as PVP $(10 \mathrm{wt} \%)$ : CLA (3 $\mathrm{wt} \%)$ in $n$-butanol, dried in $\mathrm{N}_{2}$ ambient at $60^{\circ} \mathrm{C}$ for $5 \mathrm{~min}$ followed by $120^{\circ} \mathrm{C}$ for $20 \mathrm{~min}$, and then irradiated by UV light $(254 \mathrm{~nm})$ for $30 \mathrm{~min}$. After the preparation, the cPVP layers were immersed in PGMEA or n-butanol, where they were not dissolved due to insolubilization and densification, indicating that the PVP layers were cross-linked.

\section{Device Fabrication}

3.1. cPVP/Pentacene (from SAP and DMP) OFET on Glass. The basic device structure of bottom gate/bottom contact (BCBG) OFETs and PVP cross-linked with poly(melamineco-formaldehyde) methylated in addition to the molecular structures of cross-linker molecules is depicted in Figure 1.

A $10 \mathrm{~mm}$ wide- $50 \mathrm{~nm}$ thickness of gold $(\mathrm{Au})$ metal was thermally evaporated in a vacuum pressure of $4 \times 10^{-4} \mathrm{~Pa}$ with deposition rate of $0.05 \mathrm{~nm} / \mathrm{s}$ through the shadow mask onto the glass substrate to form metal gate electrode. Thin film of cPVP was spun at $2000 \mathrm{rpm}$ on top of the Au electrode as a gate dielectric and then was baked at $100^{\circ} \mathrm{C} / 10 \mathrm{~min}$ and $200^{\circ} \mathrm{C} / 40 \mathrm{~min}$ in $\mathrm{N}_{2}$ ambient. Another cPVP layer was prepared and baked at $60^{\circ} \mathrm{C} / 5 \mathrm{~min}$ and $120^{\circ} \mathrm{C} / 20 \mathrm{~min}$ in $\mathrm{N}_{2}$ then irradiated by UV light $(254 \mathrm{~nm})$ for $30 \mathrm{~min}$. After that, source (S) and drain (D) gold electrodes were prepared using the same process condition for the gate $(G)$ contact on both thermal and photochemical prepared cPVP. The device channel width and length were $10 \mathrm{~mm}$ and $20 \mu \mathrm{m}$, respectively. Finally, pentacene thin films were deposited by spin-coating the soluble pentacene precursor of SAP $(1.4 \mathrm{wt} \%)$ at $800 \mathrm{rpm}$ for $1 \mathrm{~min}$ from chloroform $\left(\mathrm{CHCl}_{3}\right)$ in air and thermally converting the precursor films to pentacene by annealing the film at $160^{\circ} \mathrm{C}$ for $10 \mathrm{~min}$ in $\mathrm{N}_{2}$ ambient. The thickness of the film was $\sim 50 \mathrm{~nm}$ and colour of the film turned from red purple to blue after annealing. 


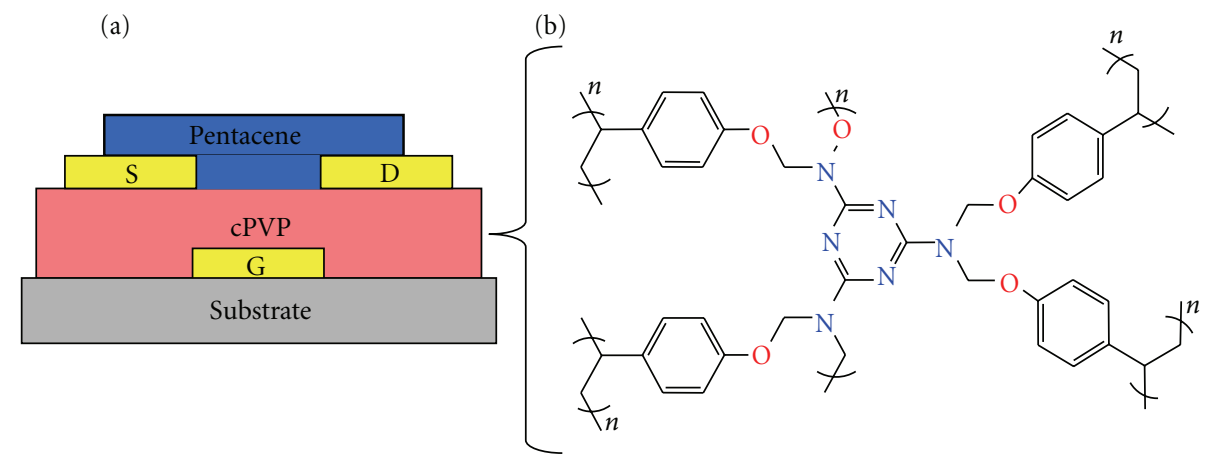

FIGURE 1: (a) Schematic diagram of an OFET and (b) the molecular structure of cross-linked PVP with poly(melamine-co-formaldehyde) methylated.

The same preparation condition used for contact electrodes with similar dimension for OFET formed from DMP precursor on heat-treated cPVP dielectric layer. Pentacene thin film $(\sim 50 \mathrm{~nm})$ was deposited by spin-coating the soluble pentacene precursor of DMP (1.4 wt\%) at $800 \mathrm{rpm}$ for $1 \mathrm{~min}$ from chloroform $\left(\mathrm{CHCl}_{3}\right)$ in air and thermally converting the precursor films to pentacene by annealing the film at $160^{\circ} \mathrm{C}$ for $30 \mathrm{~min}$ in $\mathrm{N}_{2}$ ambient. After annealing, deposited precursor turned from transparent to blue in color.

3.2. PC/Pentacene OFET on Glass. A $10 \mathrm{~mm}$ wide- $50 \mathrm{~nm}$ thickness of Au metal was thermally evaporated in a vacuum pressure of $4 \times 10^{-4} \mathrm{~Pa}$ with deposition rate of $0.05 \mathrm{~nm} / \mathrm{s}$ through the shadow mask onto the glass substrate. Chloroform solution of PC with a concentration of $1 \mathrm{wt} \%$ was spincoated at $5000 \mathrm{rpm}$ and baked for $60 \mathrm{~min}$ at $100^{\circ} \mathrm{C}$ and then cooled down to room temperature slowly. The source/drain electrodes were defined on the PC by thermally evaporating Au through shadow mask. The channel width and length were $10 \mathrm{~mm}$ and $20 \mu \mathrm{m}$, respectively. Finally pentacene active layer with thickness of $\sim 50 \mathrm{~nm}$ was deposited by thermal evaporation in vacuum of $2 \times 10^{-4} \mathrm{~Pa}$ with $50^{\circ} \mathrm{C}$ of substrate temperature and moderate deposition rate of $0.02 \mathrm{~nm} / \mathrm{s}$ for reliable thin film formation.

The thickness of the prepared thin films was measured using Sloan Dektak IIA surface profiler. The phase presence of pentacene films was studied by using SHIMADZU XD-D1 $\mathrm{X}$-ray diffractometer with graphite monochromatized $\mathrm{Cu} \mathrm{K}_{\alpha}$ radiation $(\lambda=1.54 \AA)$. Absorption spectra of pentacene films were recorded with a SHIMADZU UV-2450 UV-VIS spectrophotometer. Dielectric constants for CPVP and PC were calculated from the $C-V$ measurement using YOKOGAWAHP 4262A LCR meter. A combination of a Yamabishi and a Metronix voltage current source/monitor system was used to measure the output/transfer characteristics of the OFETs. All electrical measurements were carried out in air without any encapsulation.

\section{Results and Discussion}

4.1. Optical Analysis. The thermal conversion of SAP and DMP precursors to pentacene was analyzed by UV-vis spectroscopy. Figure 2(a) shows the absorption spectra of SAP precursor film on CPVP with absorption maximum at $257 \mathrm{~nm}$, analogues with naphthalene moiety. After heating treatment under nitrogen at $160^{\circ} \mathrm{C}$ for $10 \mathrm{~min}$, it resulted in blue colour films of pentacene. Figure 2(b) illustrates the absorption spectra of pentacene on heat-treated cPVP (red, solid line), pentacene on UV-treated cPVP (blue, short dash), both were prepared by spin-coated and pentacene on PC (brown, short dash dot) was prepared by vacuum deposition. Inset figure shows reaction scheme of spin-coated thin film of SAP to pentacene at $160^{\circ} \mathrm{C}$.

Figure 3 illustrates the absorption spectra of DMP precursor (black, solid line) and thermal pentacene (black, dot) on heat-treated cPVP in the range of $400-800 \mathrm{~nm}$. Inset shows the reaction scheme of DMP to pentacene. We observed absorption bands of DMP between 200 and $325 \mathrm{~nm}$ attributed to fine vibronic progressions. After heating the precursor at $160^{\circ} \mathrm{C}$ for $50 \mathrm{~min}$, the stronger absorption peak at $243 \mathrm{~nm}$ disappeared, while another new peak appeared at $304 \mathrm{~nm}$. From the spectra (Figures 2(b) and 3) four major peaks can be easily identified in the 500 to $700 \mathrm{~nm}$ range. There are clearly no absorption peaks in this range for an unreacted SAP and DMP precursor film and it was assumed that the samples have fully reacted.

The four major peaks located at around 542, 584, 628 , and $661 \mathrm{~nm}$ clearly indicate the characteristic bands of pentacene product from SAP, in agreement with earlier report [10]. It is well known that the pentacene exhibits main absorption peak at about $660-680 \mathrm{~nm}$ of the $S_{0}-S_{1}$ transition [14]. The pentacene film prepared on heat-treated cPVP layer shows high absorption than UV treated cPVP layer. The observed splitting peaks at about 661 and $628 \mathrm{~nm}$ are attributed to Davydov doublet of the $0-0$ band and the other two peaks at 542 and $584 \mathrm{~nm}$ to the doublet of the $0-1$ band. The vacuum deposited pentacene film gives pronounced absorption peaks than spin-coated films and a slightly larger red-shift [15] of the Davydov splitting of lower level of $0-0$ band. Similarly pentacene from heated DMP precursor shows Davydov doublet of 0-0 and 0-1 bands redshifted to about 672 and $633 \mathrm{~nm}$, respectively. The calculated values of Davydov splitting for pentacene from SAP, DMP, and vacuum deposition were $\sim 800, \sim 911$, and $\sim 919 \mathrm{~cm}^{-1}$, 


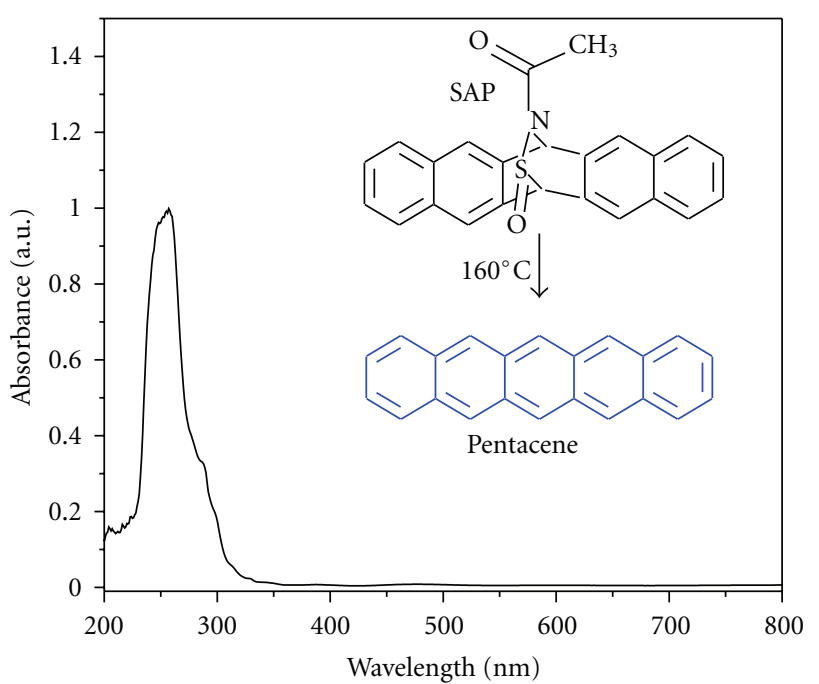

— Pristine SAP precursor on cPVP

(a)

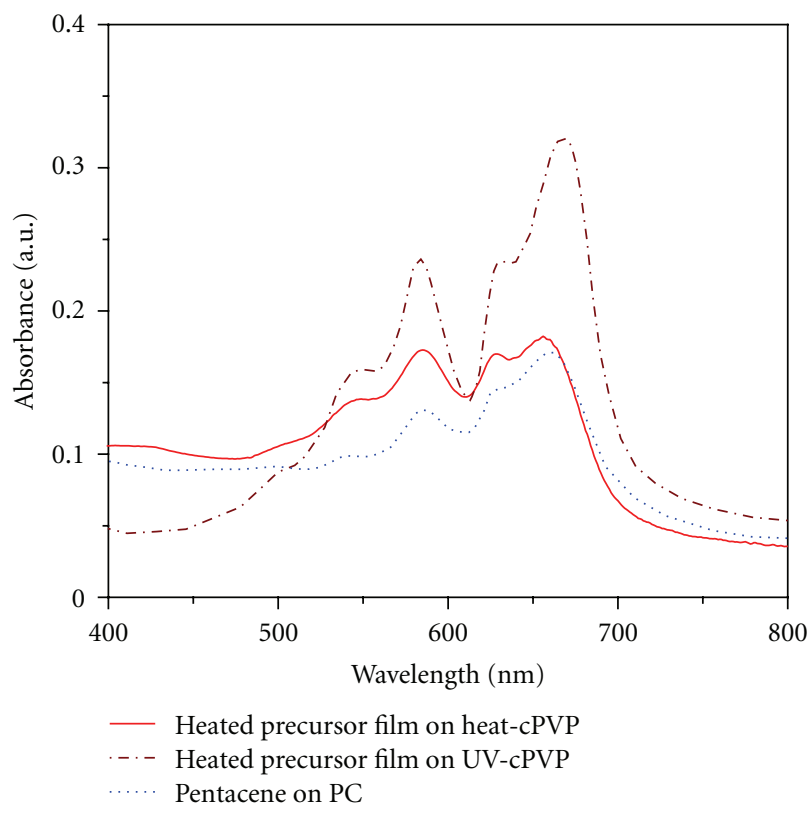

(b)

FIGURE 2: UV-vis spectra of thin films of (a) pristine SAP precursor and (b) pentacene thermally formed from SAP precursor and pentacene on PC by vacuum deposition.

respectively. This may be attributed to the presence of quasiamorphous phase in the film prepared from SAP and thinfilm phase in the films prepared from DMP and vacuum deposition [16].

4.2. Structural Analysis. In order to determine the crystalline structure of the films, XRD spectra of spin-coated pentacene from SAP precursor films were shown in Figures 4(a)-4(c). The diffraction pattern of the films was measured with Xray radiation of wavelength of $1.54 \AA$. The unheated SAP precursor (Figure 4(a)) reveals no diffraction peak, which

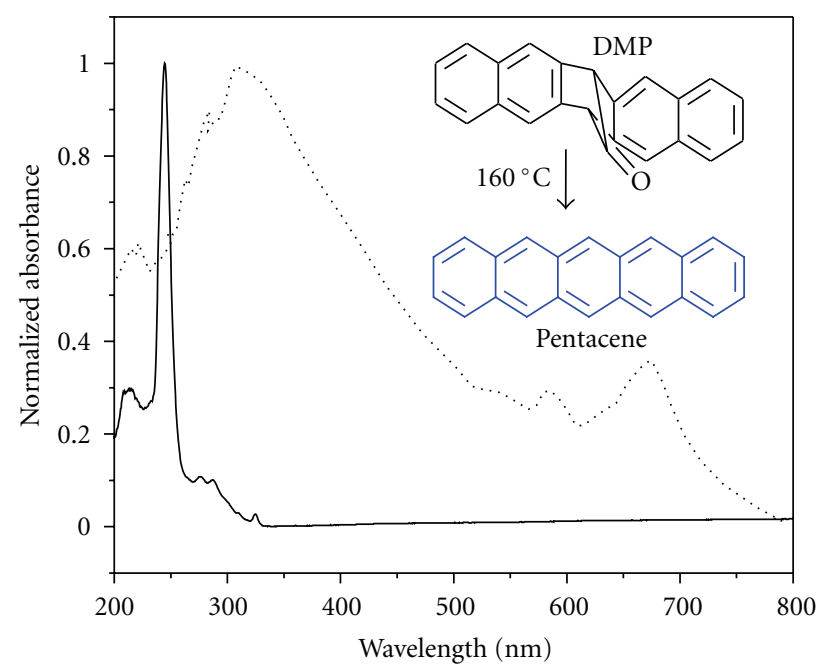

FIgURE 3: UV-vis spectra of thin films of pristine DMP precursor (black, solid line) and pentacene formed from heat-treated DMP (black, dot) on cPVP.

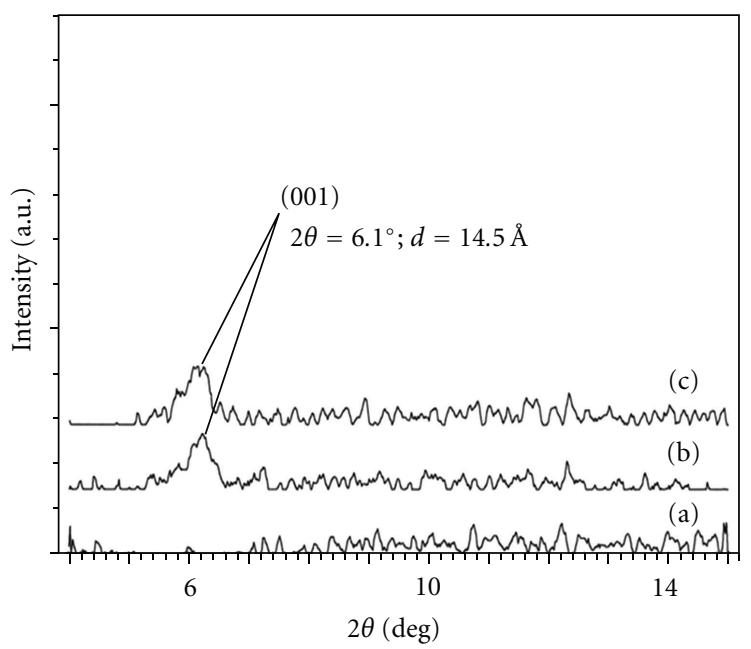

FIGURE 4: X-ray diffraction patterns of a $50 \mathrm{~nm}$ pentacene from SAP precursor of (a) untreated, (b) heat treated, and (c) UV treated on cPVP dielectric layer.

indicates that it has been formed as amorphous phase. On the contrary, the heated SAP films on both heat-cPVP and UV-cPVP exhibit broad and weak (001) diffraction peaks as depicted in Figures 4(b) and 4(c). Based on the diffraction peak at $6.1^{\circ}$, we found the $d$-spacing was to be $14.5 \AA$, which was close to that of bulk-phase and different from that of thin-film phase $[17,18]$.

The broad peak and low signal to noise ratio indicate that the deposited films were highly disordered. It suggests that the pentacene molecules may not be substantially aligned on the dielectric layers along with the certain direction and structural defects such as voids, and dislocations would be created, which are detrimental to charge transport properties of OFETs. 


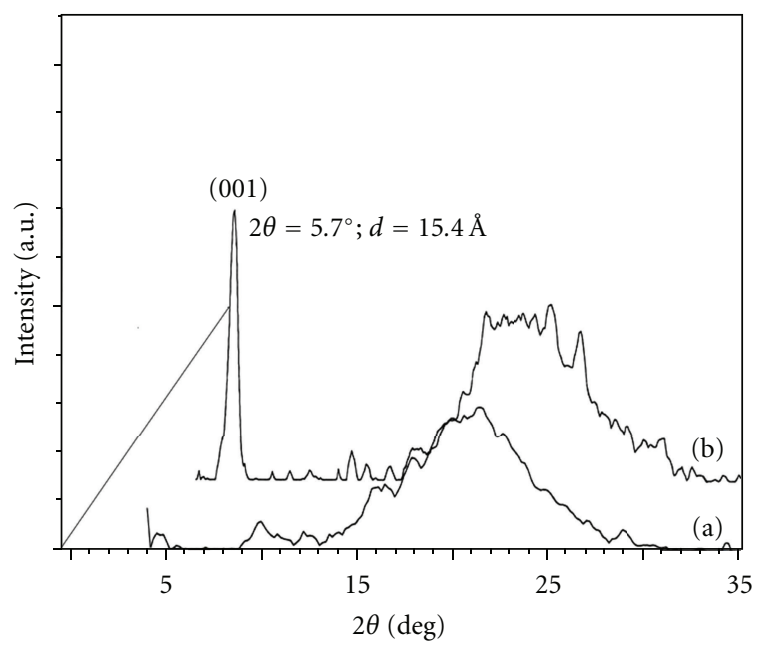

Figure 5: X-ray diffraction patterns of a $50 \mathrm{~nm}$ pentacene from DMP precursor of (a) untreated, (b) heat treated on cPVP dielectric layer.

Figure 5(a) shows XRD spectra of DMP precursor on heat-cPVP layer and the origin of the XRD profiles is shifted for a better view. The pentacene film from the DMP precursor on heat-cPVP layer (Figure 5(b)) exhibits a strong (001) diffraction peak at $5.7^{\circ}$ and corresponding $d$-spacing of $15.4 \AA$, which can reveal that the pentacene films have "thinfilm phase."

It clearly shows more ordered state of pentacene molecules and is consistent with earlier reports [17, 19]. Interestingly, the measured $d$-space value is slightly lower than the length of a single pentacene molecule (16 $\AA$ ). This suggests that pentacene molecules are slightly tilted at an angle of about $17^{\circ}$ from the normal surface of the heat-cPVP layer and hence formed layer structure. The higher order of crystalline microstructure in the film, as deduced from (001) Bragg reflection, resulted from a strong intermolecular interaction of $\pi-\pi^{*}$ electron system of the pentacene molecules within the solid film, as can be seen from the enlarged Davydov splitting in absorption spectra.

4.3. Electrical Characteristics. We now investigate the electrical performances of the pentacene OFETs based on CPVP and PC dielectric layers. PVP layer with cross-linking agent helps to reduce leakage current subsequently on/off ratio of the OFETs [20]. The cPVP as the dielectric of the OFETs may provide more stable device characteristics. The thicknesses of the cPVP and PC layers were $600 \mathrm{~nm}$ and $900 \mathrm{~nm}$, respectively. The thickness of the dielectric layers is greater than one order of magnitude of pentacene layer. It is thus suggested that thin dielectric layer controls the electrical shorting between source-drain electrodes and gate electrodes in the OFET circuit. The measured dielectric constants $(k)$ of cPVP and PC were 3.5 and 3, respectively. Figure 6 shows the output and transfer characteristics of the various OFET devices (with a channel width of $10 \mathrm{~mm}$ and a channel length of $20 \mu \mathrm{m})$.
The drain current $\left(I_{\mathrm{DS}}\right)$-drain voltage $\left(V_{\mathrm{DS}}\right)$ characteristics of the pentacene OFET fabricated by spin-coating on heat-cPVP and by vacuum deposited on PC dielectric layer are shown in Figures $6(\mathrm{a})-6(\mathrm{c})$. Here $I_{\mathrm{DS}}-V_{\mathrm{DS}}$ plots were obtained with varying the gate voltage $\left(V_{\mathrm{GS}}\right)$ in a step of $-10 \mathrm{~V}$. All devices presented here exhibit $p$-type characteristics. The transfer characteristics of above three OFETs at a fixed $V_{\mathrm{DS}}$ of $-60 \mathrm{~V}$ are depicted in Figures 6(d)-6(f). From Figure 6(d) the field effect mobility for pentacene from SAP precursor calculated in the saturation regime was $0.031 \mathrm{~cm}^{2} / \mathrm{Vs}$ and on/off current ratio of $10^{3}$. The threshold voltage $\left(V_{\text {th }}\right)$ was estimated to be $-12.5 \mathrm{~V}$. It is well known that field effect mobility is very sensitive to structure imperfections in the film. The less mobility attributed to the active layer may contain structural defects such as dislocations and voids which affect the charge carrier. This might be the reason for lower field effect mobility of OFET formed from SAP precursor. This result was supported by UV-vis and XRD spectra.

The OFET with the DMP precursor revealed relatively higher field effect mobility and lower threshold voltage. The maximum field effect mobility in the saturation regime (Figure 6(e)) was $0.09 \mathrm{~cm}^{2} / \mathrm{Vs}$ and $V_{\text {th }}$ was estimated to be $-5 \mathrm{~V}$. The on/off current ratio is $10^{3}$. Chen et al. have reported that the field effect mobility in pentacene OFET produced by spin -coating DMP precursor and then heating at $160^{\circ} \mathrm{C}$ for 1 hour is $0.0088 \mathrm{~cm}^{2} / \mathrm{Vs}$ and the value of $V_{\text {th }}$ was about $-21 \mathrm{~V}$ on heavily $\mathrm{n}$-doped Si substrate [21]. This clearly indicates that cPVP layer improved the transfer characteristics of the OFET device. Pentacene OFET on PC dielectric layer by vacuum deposition shows that the fairly large mobility value at saturation regime was $0.62 \mathrm{~cm}^{2} / \mathrm{Vs}$ as shown in Figure 6(f). The on/off ratio and $V_{\text {th }}$ were estimated to be $10^{2}$ and $-8.5 \mathrm{~V}$. An adverse effect of on/off ratio that was obtained for film deposited by vacuum suggests good crystallinity of the pentacene may also increase the conductivity of the film in the "off" state [22]. The relatively high field effect mobility may be due to not only grain size of pentacene but also microscopic molecular structures which played a significant role in the devices. The efficient charge transport properties can be attributed to increased intermolecular interactions between the molecules sensitive to molecular packing and the minimized molecular reorganization energy [23] in pentacene OFETs.

\section{Conclusions}

We have fabricated pentacene organic field effect transistors with two different polymer gate dielectric materials and obtained devices with large field effect mobility and low threshold voltage. Pentacene active layer OFET devices were successfully developed by spin-coating technique using two types of pentacene precursors. Field effect mobility of $0.09 \mathrm{~cm}^{2} / \mathrm{Vs}$ and low threshold voltage of $-5 \mathrm{~V}$ were obtained for OFET formed from 6,13-Dihydro-6,13methanopentacene-15-one precursor with thermal treatment on cross-linked poly(4-vinylphenol) dielectric layer. 


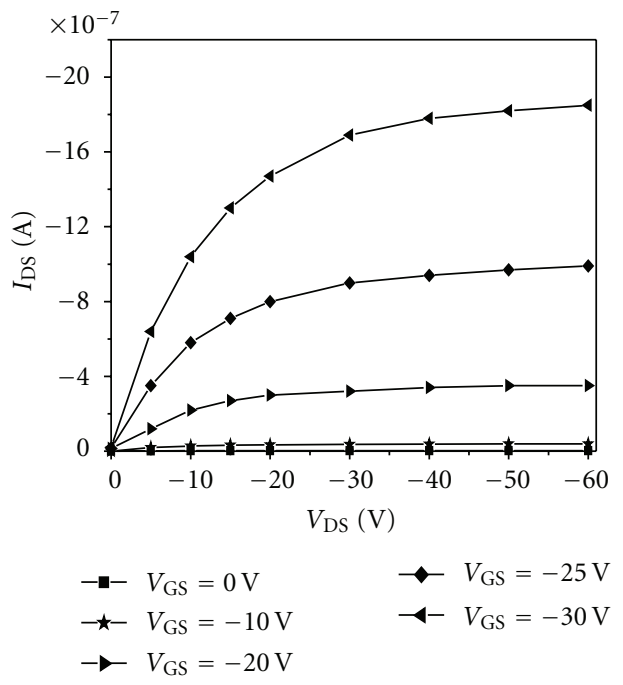

(a)

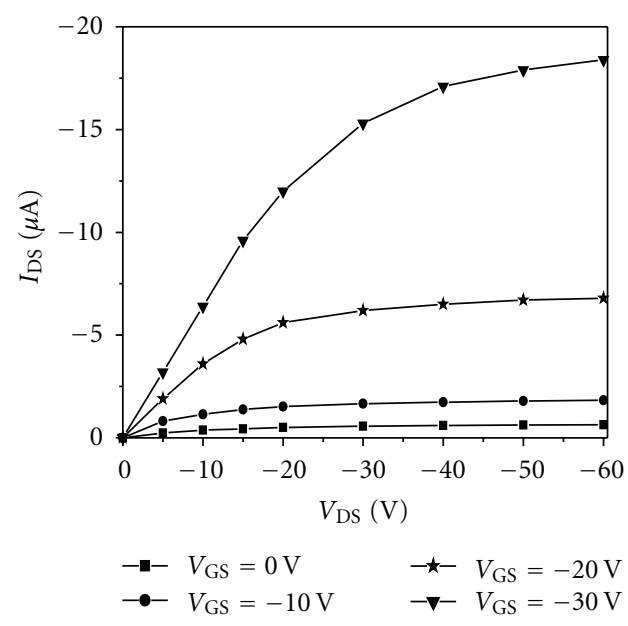

(c)

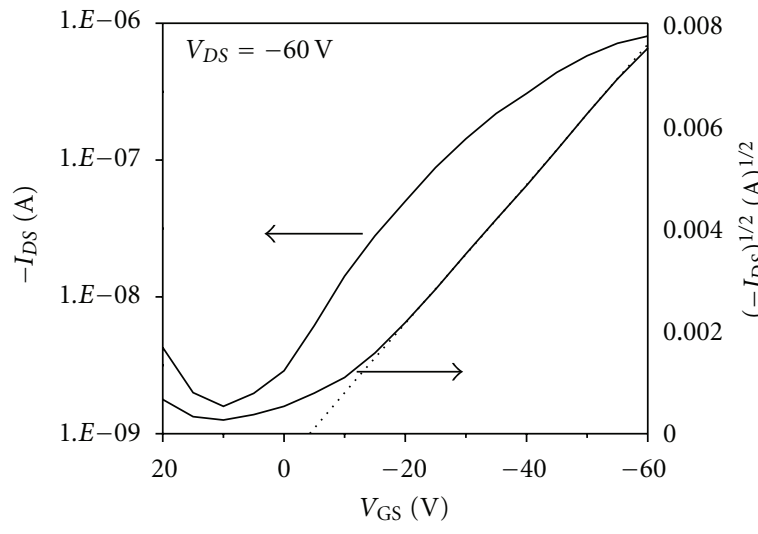

(e)

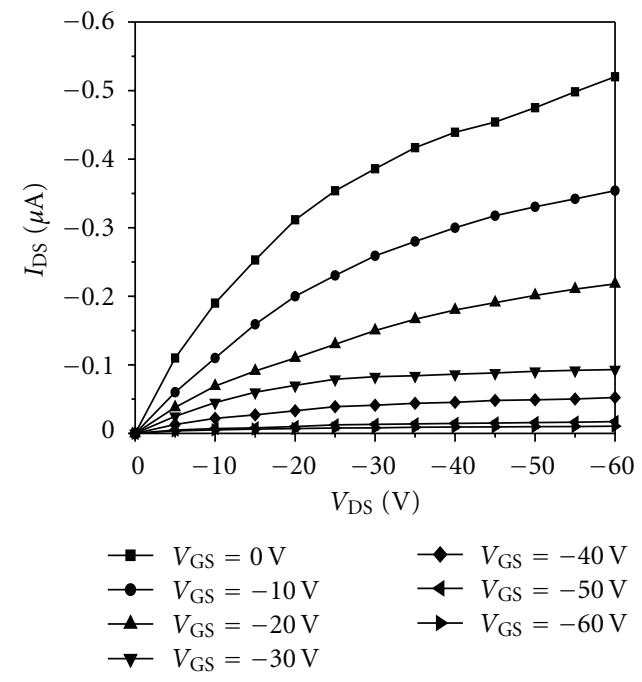

(b)

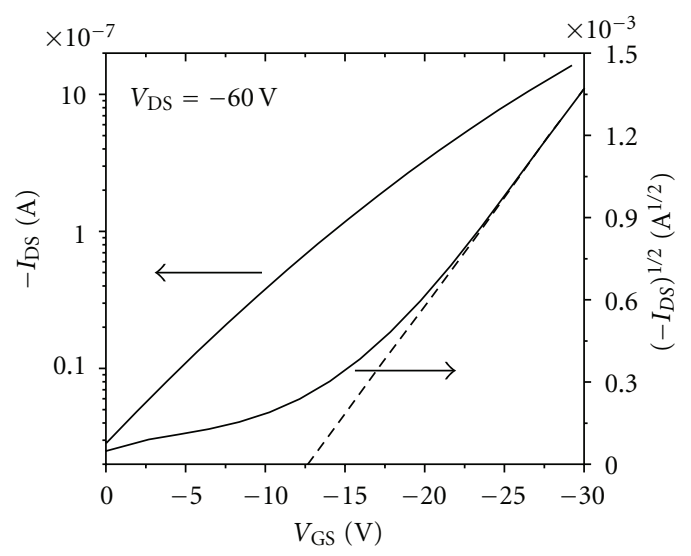

(d)

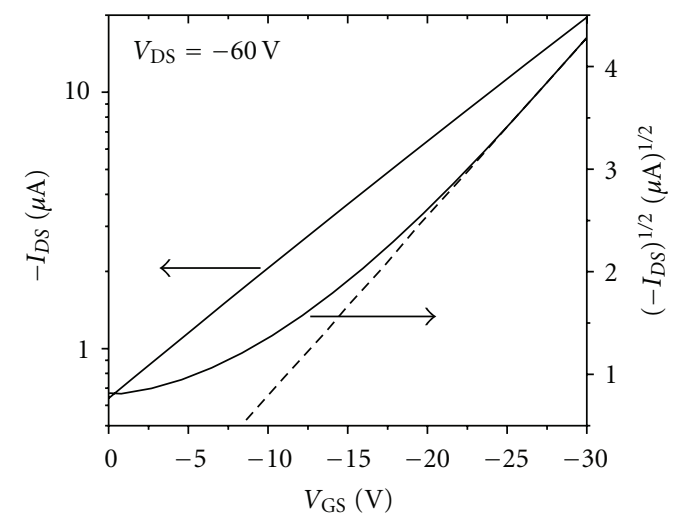

(f)

FIGURE 6: Drain current, $I_{D}$, versus drain voltage, $V_{D}$, as a function of gate voltage, $V_{G}$, for an OFET with pentacene formed (a) from heattreated SAP, (b) from heat-treated DMP precursors, and (c) by vacuum deposition. Channel width of $10 \mathrm{~mm}$ and channel length of $20 \mu \mathrm{m}$ were defined by gold source and drain electrodes. (d), (e), and (f) are plots of $I_{D}$ versus $V_{G}$ for $V_{D}=-60 \mathrm{~V}$ and ID ID $^{1 / 2}$ versus $V_{G}$ in the saturation regime for the devices in (a), (b), and (c). 
The charge carrier transport properties are influenced by strong intermolecular interaction between molecules and homogeneous molecular structure due to low reorganization energy that has been discussed. A higher mobility of $0.62 \mathrm{~cm}^{2} / \mathrm{Vs}$ and low threshold voltage of $-8.5 \mathrm{~V}$ obtained for OFET were prepared by vacuum deposition on polycarbonate dielectric layer. This study indicates that use of polymer gate dielectrics in the fabrication of OFETs offer simple processing and cost-effective with improved electrical performance. It was demonstrated that the spin-coated thin film using the soluble precursor could be a promising technology for low-cost, large area, and low temperature processing pentacene OFETs.

\section{Acknowledgments}

This research was partly supported by (Chubu Science and Technology Center Overseas researcher invitation support of NAGOYA environmental field), MEXT Private University Project Grant under Contract no. S1001033, JST Adaptable and Seamless Technology transfer Program through targetdriven R\&D no. AS232Z02610B and Joint research between AIT, and NIPPON DENWA SHISETSU.

\section{References}

[1] C. D. Dimitrakopoulos and P. R. L. Malenfant, "Organic thin film transistors for large area electronics," Advanced Materials, vol. 14, no. 2, pp. 99-107, 2002.

[2] P. Peumans, A. Yakimov, and S. R. Forrest, "Small molecular weight organic thin-film photodetectors and solar cells," Journal of Applied Physics, vol. 93, no. 7, pp. 3693-3723, 2003.

[3] Q. Zhang, S. Ochiai, G. Sawa et al., "Copper phthalocyanine thin-film transistor with a polycarbonate gate dielectric layer," Journal of the Vacuum Society of Japan, vol. 50, no. 3, pp. 155157, 2007.

[4] X. H. Zhang, S. P. Tiwari, S. J. Kim, and B. Kippelen, "Lowvoltage pentacene organic field-effect transistors with high$\kappa \mathrm{HfO}_{2}$ gate dielectrics and high stability under bias stress," Applied Physics Letters, vol. 95, no. 22, Article ID 223302, 2009.

[5] K. Suganuma, S. Watanabe, T. Gotou, and K. Ueno, "Fabrication of transparent and flexible organic field-effect transistors with solution-processed graphene source-drain and gate electrodes," Applied Physics Express, vol. 4, no. 2, Article ID 021603, 2011.

[6] T. B. Singh, N. Marjanović, G. J. Matt, N. S. Sariciftci, R. Schwödiauer, and S. Bauer, "Nonvolatile organic field-effect transistor memory element with a polymeric gate electret," Applied Physics Letters, vol. 85, no. 22, article no. 3, pp. 5409$5411,2004$.

[7] H. L. Cheng, Y. S. Mai, W. Y. Chou, L. R. Chang, and X. W. Liang, "Thickness-dependent structural evolutions and growth models in relation to carrier transport properties in polycrystalline pentacene thin films," Advanced Functional Materials, vol. 17, no. 17, pp. 3639-3649, 2007.

[8] H. Klauk, M. Halik, U. Zschieschang, G. Schmid, W. Radlik, and W. Weber, "High-mobility polymer gate dielectric pentacene thin film transistors," Journal of Applied Physics, vol. 92, no. 9, pp. 5259-5263, 2002.

[9] T. F. Guo, Z. J. Tsai, S. Y. Chen, T. C. Wen, and C. T. Chung, "Influence of polymer gate dielectrics on n-channel conduction of pentacene-based organic field-effect transistors," Journal of Applied Physics, vol. 101, no. 12, Article ID 124505, 2007.

[10] A. Afzali, C. D. Dimitrakopoulos, and T. L. Breen, "Highperformance, solution-processed organic thin film transistors from a novel pentacene precursor," Journal of the American Chemical Society, vol. 124, no. 30, pp. 8812-8813, 2002.

[11] H. Uoyama, H. Yamada, T. Okujima, and H. Uno, "Pentacene precursors for solution-processed OFETs," Tetrahedron, vol. 66, no. 34, pp. 6889-6894, 2010.

[12] S. Uppalapati, S. Chada, M. H. Engelhard, and M. Yan, "Photochemical reactions of poly(4-vinylphenol) thin films," Macromolecular Chemistry and Physics, vol. 211, no. 4, pp. 461-470, 2010.

[13] T. B. Singh, F. Meghdadi, S. Günes et al., "High-performance ambipolar pentacene organic field-effect transistors on poly(vinyl alcohol) organic gate dielectric," Advanced Materials, vol. 17, no. 19, pp. 2315-2320, 2005.

[14] D. Faltermeier, B. Gompf, M. Dressel, A. K. Tripathi, and J. Pflaum, "Optical properties of pentacene thin films and single crystals," Physical Review B, vol. 74, no. 12, Article ID 125416, 2006.

[15] S. Köstler, A. Rudorfer, A. Haase, V. Satzinger, G. Jakopic, and V. Ribitsch, "Direct condensation method for the preparation of organic-nanoparticle dispersions," Advanced Materials, vol. 21, no. 24, pp. 2505-2510, 2009.

[16] O. Ostroverkhova, S. Shcherbyna, D. G. Cooke et al., "Optical and transient photoconductive properties of pentacene and functionalized pentacene thin films: dependence on film morphology," Journal of Applied Physics, vol. 98, no. 3, Article ID 033701, pp. 1-12, 2005.

[17] C. D. Dimitrakopoulos, A. R. Brown, and A. Pomp, "Molecular beam deposited thin films of pentacene for organic field effect transistor applications," Journal of Applied Physics, vol. 80, no. 4, pp. 2501-2508, 1996.

[18] D. Knipp, R. A. Street, A. Völkel, and J. Ho, "Pentacene thin film transistors on inorganic dielectrics: morphology, structural properties, and electronic transport," Journal of Applied Physics, vol. 93, no. 1, pp. 347-355, 2003.

[19] H. L. Cheng, Y. S. Mai, W. Y. Chou, and L. R. Chang, "Influence of molecular structure and microstructure on device performance of polycrystalline pentacene thin-film transistors," Applied Physics Letters, vol. 90, no. 17, Article ID 171926, 2007.

[20] S. C. Lim, S. H. Kim, J. B. Koo et al., "Hysteresis of pentacene thin-film transistors and inverters with cross-linked poly(4vinylphenol) gate dielectrics," Applied Physics Letters, vol. 90, no. 17, Article ID 173512, 2007.

[21] K. Y. Chen, H. H. Hsieh, C. C. Wu, J. J. Hwang, and T. J. Chow, "A new type of soluble pentacene precursor for organic thinfilm transistors," Chemical Communications, no. 10, pp. 10651067, 2007.

[22] M. H. Choo, J. H. Kim, and S. Im, "Hole transport in amorphous-crystalline-mixed and amorphous pentacene thin-film transistors," Applied Physics Letters, vol. 81, no. 24, pp. 4640-4642, 2002.

[23] N. E. Gruhn, D. A. Da Silva Filho, T. G. Bill et al., "The vibrational reorganization energy in pentacene: molecular influences on charge transport," Journal of the American Chemical Society, vol. 124, no. 27, pp. 7918-7919, 2002. 

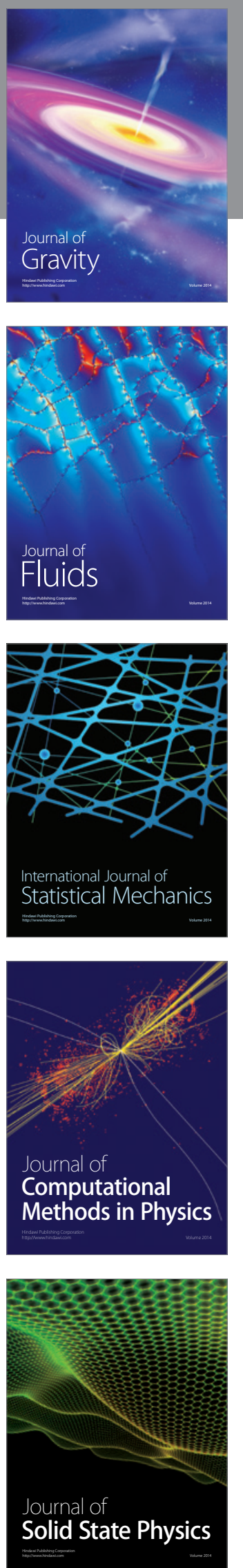
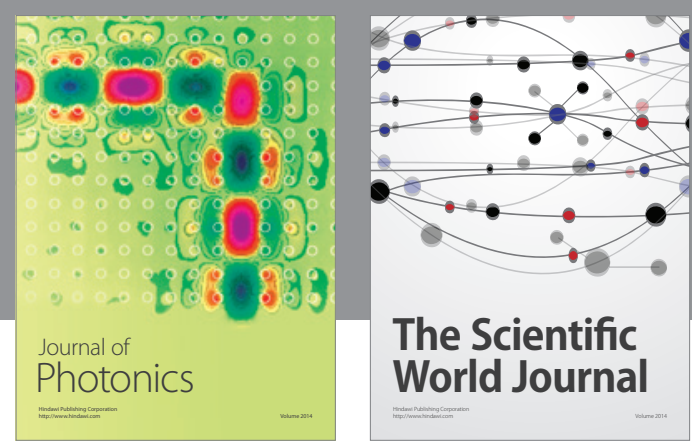

The Scientific World Journal

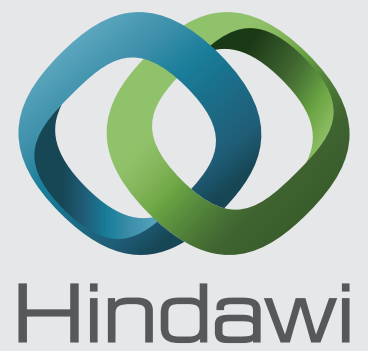

Submit your manuscripts at http://www.hindawi.com
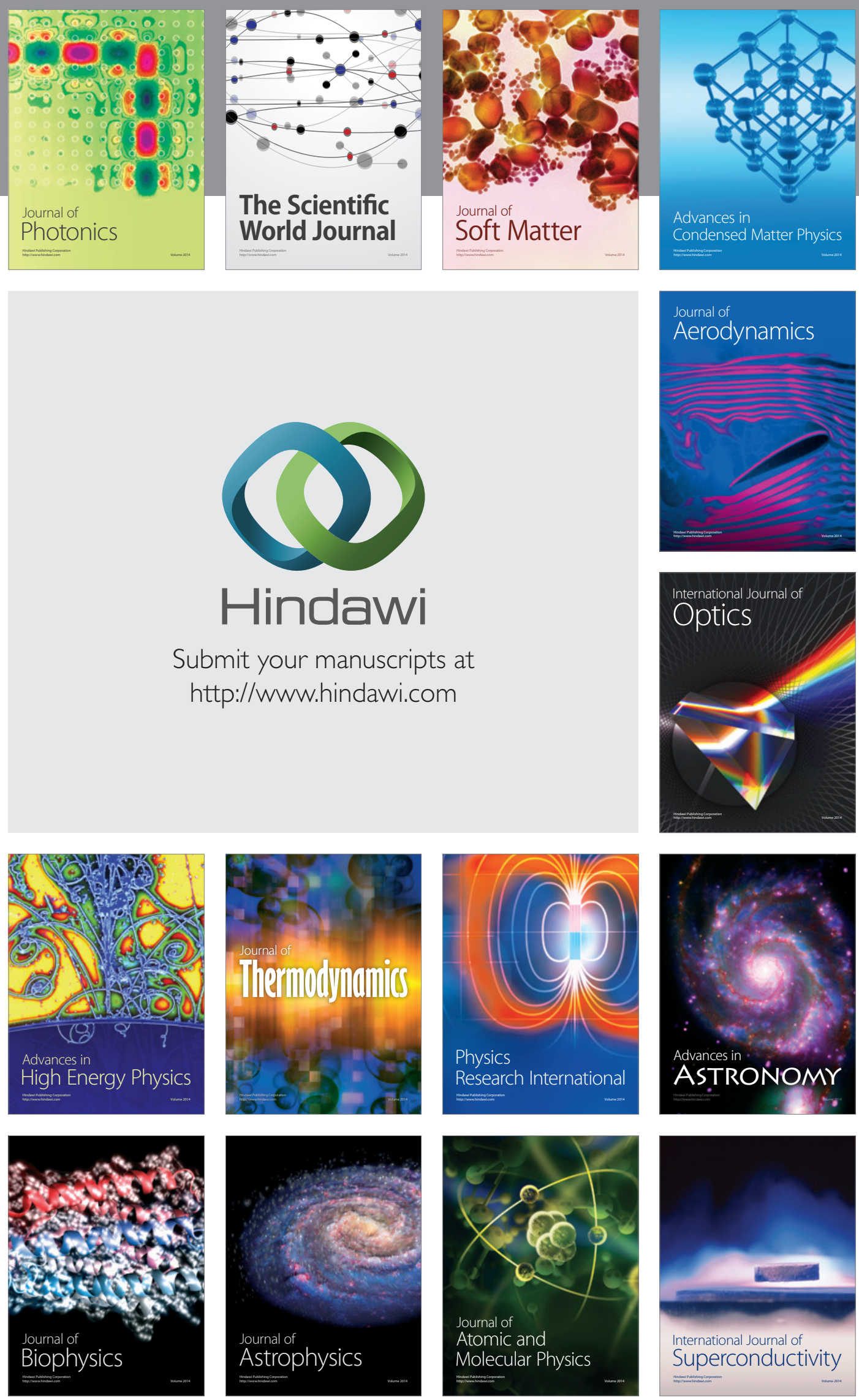
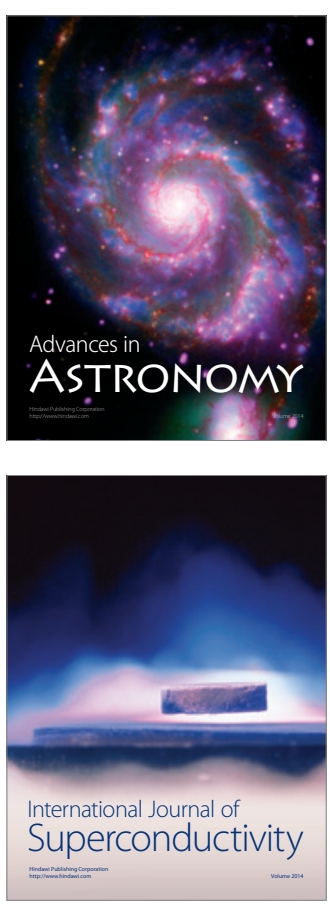